\title{
Polyvinylamine-Streptavidin Complexes Labeled with a Europium Chelator: A Universal Detection Reagent for Solid-Phase Time Resolved Fluorometric Applications
}

\author{
ANDREAS SCORILAS and ELEFTHERIOS P. DIAMANDIS \\ Department of Laboratory Medicine and Pathobiology, University of Toronto, Toronto, Ontario, \\ Canada, and Department of Pathology and Laboratory Medicine, Mount Sinai Hospital, Toronto, \\ Ontario, Canada
}

\begin{abstract}
Objectives: To describe the synthesis and characteristics of a new streptavidin-based universal detection reagent which is multiply labeled with the europium chelate of 4,7-bis(chlorosulfophenyl)1,10-phenanthroline-2,9-dicarboxylic-acid (BCPDA).

Methods and results: Polyvinylamine (PVA) was first labeled with biotin(b) and then with BCPDA to create (b) $x$-PVA-(BCPDA) . By mixing controlled amounts of this complex with streptavidin (SA) and a fixed amount of $\mathrm{Eu}^{3+}$, we were able to produce the conjugate $(\mathrm{SA})_{\mathrm{z}}-(\mathrm{b})_{\mathrm{x}}-\mathrm{PVA}(\mathrm{BCPDA})_{\mathrm{y}}-\mathrm{Eu}^{3+}$. This conjugate is reactive, highly fluorescent, and stable for at least 12 months. It was used to develop model solid-phase time-resolved fluoroimmunoassays for biotinylated mouse $\lg \mathrm{G}$ and prostate spesific antigen (PSA). Detection limits achieved were around 1 to $2 \mathrm{ng} / \mathrm{L}\left(\sim 3 \times 10^{-18} \mathrm{moles} /\right.$ assay).

Conclusions: A new universal detection reagent was synthesized, which can be used in combination with biotinylated reagents (e.g., antibodies, DNA probes, etc.) for the development of highly sensitive solid-phase time-resolved fluorescence-based assays. Copyright @ 2000 The Canadian Society of Clinical Chemists
\end{abstract}

KEY WORDS: BCPDA; PVA; biotin; streptavidin; afetoprotein; PSA; NHS-LC-LC-biotin; time-resolved fluoroimmunoassay.

\section{Introduction}

$\mathbf{C}$ Yonventional fluorescein and rhodamine derivatives have been used in the past as immunological labels with a maximum attainable sensitivity of $\sim 10^{-9}-10^{-10} \mathrm{M}$ (1). Scattering interferences are aggrevated by the small Stokes shifts $(24-50 \mathrm{~nm})$ of conventional fluorophores. In addition, the inner filter effect as well as the background fluorescence signals from biological samples, cuvettes, solvents, optics, etc. usually arise between 350 and $600 \mathrm{~nm}$,

Correspondence: E.P. Diamandis, Department of Pathology and Laboratory Medicine, Mount Sinai Hospital, 600 University Avenue, Toronto, Ontario M5G 1X5, Canada. Fax: (416) 586-8628. E-mail: ediamandis@mtsinai. on.ca

Manuscript received February 6, 2000; revised April 20, 2000; accepted May 3, 2000. and overlap extensively with the emission spectra of many of these fluorophores (2).

Fluorescent europium chelates exhibit large Stokes shifts $(\sim 290 \mathrm{~nm})$ with no overlap between the excitation and emission spectra. These chelates have very narrow emission bands (10-nm bandwidth) at $615 \mathrm{~nm}$, which do not overlap with serum native fluorescence. In addition, their long fluorescence lifetimes $(600-1000 \mu \mathrm{s})$ allow use of microsecond time-resolved fluorescence measurements, which further reduce the observed background signals $(2-5)$.

Time-resolved fluoroimmunoassays (TR-FIA) using europium and other lanthanide complexes as fluorescent labels have attracted wide interest (28). $\mathrm{A} \mathrm{Eu}^{3+}$ chelator, 4,7-bis(chlorosulfophenyl)-1,10phenanthroline-2,9-dicarboxylic acid (BCPDA) has been synthesized and extensively used $(4,9)$. Another $\mathrm{Eu}^{3+}$ chelator, $4,4^{\prime}$-bis $\left(1^{\prime \prime}, 1^{\prime \prime}, 1^{\prime \prime}, 2^{\prime \prime}, 2^{\prime \prime}, 3^{\prime \prime}, 3^{\prime \prime}\right.$-heptafluoro-4", 6"-hexanedion-6"-yl)chlorosulfo-o-terphenyl (BHHCT) was recently reported (6).

In this article, we describe the synthesis of a streptavidin-based universal detection reagent that is suitable for highly sensitive, solid-phase timeresolved fluorometric applications. This reagent is superior in sensitivity to conjugates reported previously (10) and, in addition, is stable on storage and can be prepared with a simple and reproducible procedure. This new detection reagent may have diverse applications in immunoassays, DNA-based assays, cell analysis and microarrays.

\section{Experimental section}

\section{Materials}

The $\mathrm{Eu}^{3+}$ chelator 4,7-bis(chlorosulfophenyl)1,10-phenanthroline-2,9-dicarboxylic acid (BCPDA) was synthesized as described previously (9). Affinity purified streptavidin (SA) was purchased from Sigma Chemical Company (St. Louis, MO, USA). 
Sulfosuccinimidyl 6-(biotinamido) hexanoate(SulfoNHS-LC-LC-Biotin) was obtained from Pierce Chemical Company (Rockford, IL, USA). Polyvinylamine hydrocloride was obtained from Chemical Dynamics Corp. (South Plainfied, NJ, USA); the average molecular weight of PVA was $\sim 100,000$ (molecular weight distribution 50,000-150,000). Only a single lot of this reagent was available. For this reason, lot-to-lot variation was not examined. All other chemicals used were from Sigma unless otherwise stated. Microfluor, white, opaque 96-well microtiter plates were purchased from Greiner Labortechnik (Germany).

\section{INSTRUMENTATION}

For measurement of solid-phase fluorescence, the CyberFluor 615 immunoanalyzer, a time-resolved fluorometer with a nitrogen laser as the excitation source was used (MDS Nordion, Kanata, Ontario, Canada). This instrument is capable of measuring fluorescence at the bottom of white microtitration wells with a total measurement time of 1 seconds/ well. Selected measurements were further performed on the instrument 'Analyst' by LJL Biosystems (Sunnyvale, CA, USA). Because the data obtained were identital with the two instruments, only measurements with the CyberFluor 615 immunoanalyzer will be presented here. UV-absorption spectra were recorded on a HP Model 8450A diode array spectrophotometer (Hewlett-Packard Canada, Ontario, Canada). High performance liquid chromatography (HPLC) was carried out using a BioSil TSK-250 size exclusion column from Bio-Rad Laboratories (Richmond, CA, USA) on a Waters HPLC system (Millipore Canada, Mississauga, Ontario, Canada).

\section{Preparation of Biotin-PVA-BCPDA COMPLEX}

We prepared a $10 \mathrm{mg} / \mathrm{mL}$ stock solution of polyvinylamine hydrochloride (PVA) in a $0.5 \mathrm{M}$ carbonate buffer, pH 9.1. In $200 \mu \mathrm{L}(2 \mathrm{mg} ; \sim 20 \mathrm{nmol})$ of the PVA solution we then added $200 \mu \mathrm{g}(\sim 300 \mathrm{nmol})$ of NHS-LC-LC biotin dissolved in $20 \mu \mathrm{L}$ of carbonate buffer and incubated at room temperature for 1 hour [molar ratio of PVA: biotin $-1: 15]$. We then added more $0.5 \mathrm{M}$ carbonate buffer, $\mathrm{pH} 9.1$, to adjust volume to $1 \mathrm{~mL}$. Solid BCPDA was pulverized to a fine powder. To the PVA solution, we added $5 \mathrm{mg}$ of solid BCPDA and incubated at room temperature with vigorous shaking. We repeated this step another three times (total amount of BCPDA added was 20 $\mathrm{mg}$ or $\sim 40 \mu \mathrm{mol})$. Once the solution clarified $(\sim 3-4$ $\mathrm{h}$ total incubation time), it was transferred to dialysis tubing (molecular weight cutoff $\sim 12,000$ ) and dialyzed in $5 \mathrm{~L}$ of $0.1-\mathrm{M} \mathrm{NaH} \mathrm{CO}_{3}$ solution overnight. Dialysis was repeated at least another two times to remove as much as possible of non-reacted biotin and BCPDA.
Purification of Biotinylated and BCPDA-Labeled PVA BY HPLC

In order to remove quantitatively loosely bound BCPDA, we used gel filtration HPLC. The dialyzed preparation was concentrated by centrifugation to 0.3-0.5 mL using Amicon microconcentration units (M.W. 30,000 cutoff). The HPLC was run isocratically at a flow rate of $0.8 \mathrm{~mL} / \mathrm{min}$, with a mobile phase of $0.05 \mathrm{M}$ Tris buffer, $\mathrm{pH}$ 7.70. Absorbance was monitored at $325 \mathrm{~nm}$ (absorption maximum of BCPDA). We used a silica-based TSK-250 gel filtration column. After injecting the whole conjugate, we collected and pooled the fractions containing biotinylated PVA-BCPDA (fractions 11-15; $4 \mathrm{~mL}$ ). These fractions were identified by the specific absorbance of BCPDA and by using an assay that specifically measures biotinylated PVA-BCPDA. This assay used streptavidin-coated microtiter plates. HPLC fractions $\left(10 \mu \mathrm{L}\right.$ of each plus $\left.90 \mu \mathrm{L} \mathrm{H} \mathrm{H}_{2} \mathrm{O}\right)$ were added to the plate, incubated for $30 \mathrm{~min}$ and then washed. We then added a $10^{-5} \mathrm{M} \mathrm{EuCl}_{3}$ solution in Tris buffer for $10 \mathrm{~min}$, washed and dried the plate. Fluorescence was then measured in a time-resolved mode on the CyberFluor 615 Immunoanalyzer. With this assay, only biotinylated-PVA-BCPDA is quantified. Non-biotinylated moieties will not be captured while biotinylated moieties not containing BCPDA will be captured but will not fluoresce in the presence of $\mathrm{Eu}^{3+}$.

Using absorbance measurements, we calculated that labeled PVA contains approximately 50-100 BCPDA molecules per molecule of PVA (the extinction coefficient of BCPDA is $1.52 \times 10^{4} \mathrm{~mol}^{-1}$ L.cm ${ }^{-1}$ at $\left.325 \mathrm{~nm}\right)$. In subsequent steps, we will refer to this reagent as the (b) $)_{x}-P V A-(B C P D A)_{y}$ complex.

Preparation of Streptavidin Conjugates $\left[(\mathrm{SA})_{\mathrm{z}}-(\mathrm{B})_{\mathrm{x}^{-}}\right.$ PVA-(BCPDA) $]$

Our aim was to produce active streptavidin conjugates of biotinylated PVA-(BCPDA) $y_{y}$. We took advantage of the multiple binding sites of SA for biotin and performed this conjugation by simply mixing a controlled amount of streptavidin with an appropriate amount of (b) $)_{\mathrm{x}}$-PVA-BCPDA) $)_{\mathrm{y}}$ and $\mathrm{Eu}^{3+}$. A typical protocol is as follows: $1 \mathrm{~mL}$ of a $60 \mathrm{~g} / \mathrm{L}$ bovine serum albumin solution in $0.1 \mathrm{M}$ Tris buffer, $\mathrm{pH}$ 7.80, containing $\mathrm{EuCl}_{3}, 10^{-5} \mathrm{M}$, plus $10 \mu \mathrm{L}$ of a 1 $\mathrm{mg} / \mathrm{mL}$ solution of streptavidin in water plus $40-55$ $\mu \mathrm{L}$ of the (b) $\mathrm{x}$-PVA-(BCPDA) conjugate, prepared as described above. The exact amount of the conjugate must be determined by titration (see results section) using a model solid-phase immunoassay as an indicator (see below). The mixture is then incubated for $1 \mathrm{~h}$ at $55^{\circ} \mathrm{C}$ and is ready for use (se below). If stored at $4{ }^{\circ} \mathrm{C}$, this conjugate is stable for at least 12 months, verified by performing solid phase immunoassays without any loss of sensitivity. 


\section{EVALUATION OF CONJUGATE'S REACTIVITY}

Microtiter plates were coated overnight with varying amounts of biotinylated mouse $\operatorname{IgG}(0,0.5,5$, and $50 \mathrm{ng} / \mathrm{well} ; 100 \mu \mathrm{L} / \mathrm{well})$ after dilution in a $0.05 \mathrm{M}$ Tris buffer, $\mathrm{pH}$ 7.80. After washing, we added 100 $\mu \mathrm{L}$ of the $(\mathrm{SA})_{\mathrm{z}}-(\mathrm{b})_{\mathrm{x}}-\mathrm{PVA}-(\mathrm{BCPDA})_{\mathrm{y}}-\mathrm{Eu}^{3+}$ conjugate, diluted tenfold (diluent is a $60 \mathrm{~g} / \mathrm{L}$ bovine serum albumin solution in a $0.1 \mathrm{~mol} / \mathrm{L}$ Tris buffer, $\mathrm{pH} 7.80$ ). After incubation for $25 \mathrm{~min}$, the plate was washed and dried in a forced air microplate dryer and solid-phase fluorescence was measured with the CyberFluor 615 Time-Resolved Fluorometer. This assay was used as an indicator of conjugate's reactivity in all conjugation reactions. Optimization experiments is shown in the Results section of this article.

\section{IMMUNOASSAY FOR PROSTATE SPECIFIC ANTIGEN}

An ELISA-type sandwich PSA assay comprised of two PSA monoclonal antibodies was used. The capture antibody (coded 8301; Diagnostic Systems Laboratories, Houston, TX, USA) was immobilized onto polystyrene microtitration wells at a concentration of $0.5 \mathrm{mg} / 100 \mu \mathrm{L}$ per well in $50 \mathrm{mM}$ Tris buffer $(\mathrm{pH}$ 7.8). This incubation was performed overnight at room temperature. The wells were washed six times with wash solution $(150 \mathrm{mM} \mathrm{NaCl}, 50 \mathrm{mM}$ Tris, 1 $\mathrm{mM} \mathrm{NaN} \mathrm{N}_{3}, 0.05 \%$ Tween 20) following coating and all other incubations. PSA calibrators consisted of PSA purified from seminal plasma (a gift from Dr. Thomas Stamey, Stanford University, CA) in a bovine serum albumin (BSA) diluent (50 mM Tris, $\mathrm{pH}$ 7.8 , containing $60 \mathrm{~g} / \mathrm{L}$ BSA). The PSA calibrators were diluted to concentrations of $0,1,5,20,100,500$, 2000 , and $10,000 \mathrm{ng} / \mathrm{L}$. The $100 \mu \mathrm{L}$ of each calibrator were pipetted with $50 \mu \mathrm{L}$ of biotinylated PSA detection monoclonal antibody (coded 8311; Diagnostic Systems Laboratories) diluted 1000 -fold in assay buffer $(50 \mathrm{mmol} / \mathrm{L}$ Tris, $\mathrm{pH} 7.8,100 \mathrm{~mL} / \mathrm{L}$ goat serum, $60 \mathrm{~g} / \mathrm{L}$ BSA, $50 \mathrm{~mL} / \mathrm{L}$ mouse serum, $10 \mathrm{~g} / \mathrm{L}$ bovine immunoglobulins, $5 \mathrm{~g} / \mathrm{L}$ Tween 20, 500 $\mathrm{mmol} / \mathrm{L} \mathrm{KCl}$ ) and incubated with shaking at room temperature for $1 \mathrm{~h}$. The wells were washed six times and $100 \mu \mathrm{L}$ of tenfold diluted $(\mathrm{SA})_{\mathrm{z}}-(\mathrm{b})_{\mathrm{x}}-\mathrm{PVA}-$ $(\mathrm{BCPDA})_{\mathrm{y}}-\mathrm{Eu}^{3+}$ conjugate were added and incubated at RT for 25 min. Following six times washing and drying with a stream of cold air, the resulting fluorescence was measured on a Cyberfluor 615 Immunoanalyzer.

\section{Results}

We succeeded in developing a conjugate of streptavidin indirectly labeled with the europium chelate of BCPDA. BCPDA is covalently bound to PVA and it is linked to streptavidin via biotin interaction. The complex successfully recognizes and binds other biotinylated antibodies. In Figure 1 we present a chromatogram showing purification of the (b) $x$-PVA$(\mathrm{BCPDA})_{\mathrm{y}}$ complex from unreacted BCPDA, by us-

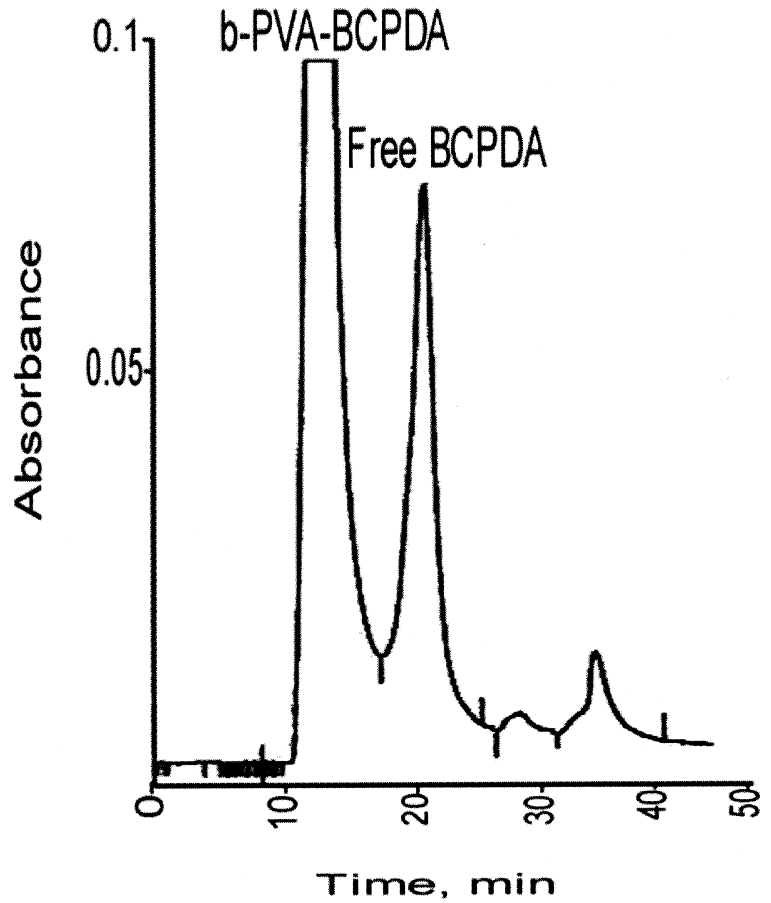

Figure 1 - Purification of the (b) $)_{x}$-PVA-(BCPDA) conjugate by HPLC on a silica-based TSK-250 gel filtration column. The HPLC was run isocratically at a flow rate of $0.8 \mathrm{~mL} / \mathrm{min}$. Absorbance was monitored at $325 \mathrm{~nm}$. For more details see text.

ing HPLC. This conjugate was further recognized by binding to streptavidin-coated microtiter plates, as described in the Methods section. The conjugate (b) x $_{\mathrm{x}} \mathrm{PVA}-(\mathrm{BCPDA})_{\mathrm{y}}$ was further linked to SA, to produce the final reagent, $(\mathrm{SA})_{\mathrm{z}}-(\mathrm{b})_{\mathrm{x}}-\mathrm{PVA}-(\mathrm{BCP}-$ $\mathrm{DA})_{\mathrm{y}}$. The critical step in the preparation of this conjugate is the ratio of SA to $(b)_{x}-P V A-(B C P D A)_{y}$. If the latter reagent is in excess, the streptavidin will be present in less than optimal concentration and, the sensitivity of the reagent will not be optimal. The best ratio of SA and (b) - PVA-(BCPDA) must be found empirically by titration, as shown in Figure 2 . In this experiment, the streptavidin amount was kept constant and the amount of the conjugate was varied. In the example of Figure 2, optimal volume of conjugate was $49 \mu \mathrm{L}$. More dilute or more concentrated solutions of the reagent can be easily prepared by simply adjusting the amount of the added reagents.

We have optimized the preparation of this reagent in terms of the following (data not shown): use of other biotinylation reagents (NHS-LC-biotin, NHSpolyethylene glycol-biotin), amount of biotinylation reagent, amount of BCPDA, mode of addition of BCPDA (solid vs. solution), various incubation times, type of BCPDA carrier (we tried a number of protein molecules in addition to PVA), temperature of conjugate formation (room temperature to $65{ }^{\circ} \mathrm{C}$ with $5{ }^{\circ} \mathrm{C}$ steps), amount of $\mathrm{Eu}^{3+}$ and type of diluent with or without $\mathrm{Eu}^{3+}$.

In Figure 3, we show the sensitivity and linearity of measuring solid phase-immobilized biotinylated 


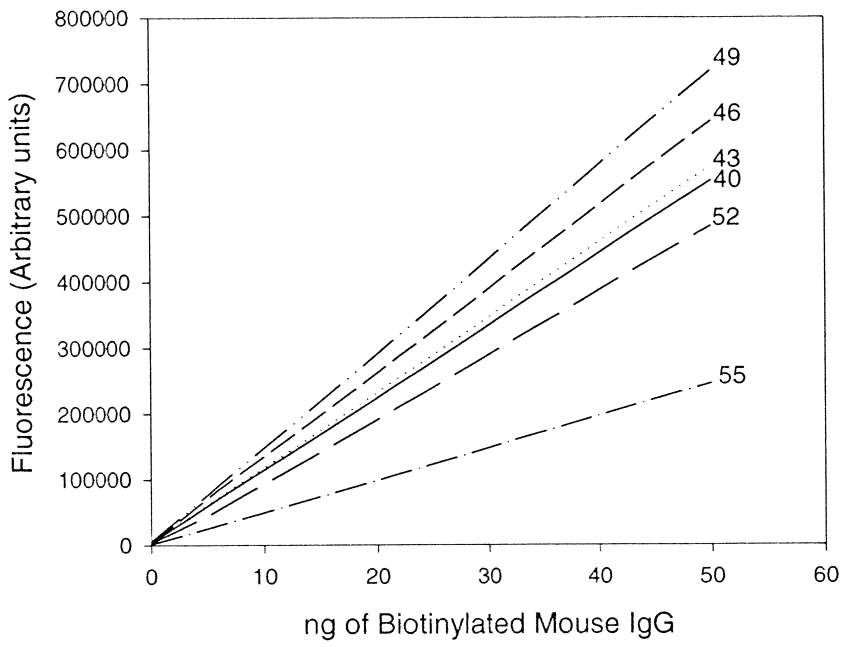

Figure 2 - Titration of streptavidin with (b) - PVA-(BCP$\mathrm{DA})_{\mathrm{y}}$ conjugate. The titration mixture contained $1 \mathrm{~mL}$ of a $60 \mathrm{~g} / \mathrm{L}$ bovine serum albumin solution in $0.1 \mathrm{M}$ Tris buffer, $\mathrm{pH} 7.80, \mathrm{EuCl}_{3}\left(10^{-5} \mathrm{M}\right), 0.01 \mathrm{mg} / \mathrm{mL}$ streptavidin, and 40 to $55 \mu \mathrm{L}$ of conjugate. Each mixture was used to detect immobilized biotinylated mouse IgG at four different concentrations: $0,0.5,5$, and $50 \mathrm{ng} / \mathrm{well}$, run in triplicate. Lines were constructed using linear regression. Optimal conjugate was formed with $49 \mu \mathrm{L}$ of conjugate.

mouse IgG. At the level of $500 \mathrm{pg} /$ assay $(\sim 3 \mathrm{fmol} /$ assay), the signal to background ratio of fluorescence is about 100 -fold. The detection limit is $\sim 10$ pg/assay and linearity is excellent. Detection limit was defined as the amount of analyte that could be distinguished from the background signal with $95 \%$ confidence (background signal plus two standard deviations).

The principle of the PSA assay used is shown in Figure 4. As shown in Figure 5, we could detect about $1 \mathrm{ng} / \mathrm{L}$ of PSA (100 fg/assay; equivalent to $3 \times 10^{-18}$ moles of PSA). Linearity extends over four decades of PSA concentration (1 to $10,000 \mathrm{ng} / \mathrm{L}$ ). Precision was $<10 \%$ at PSA concentrations between

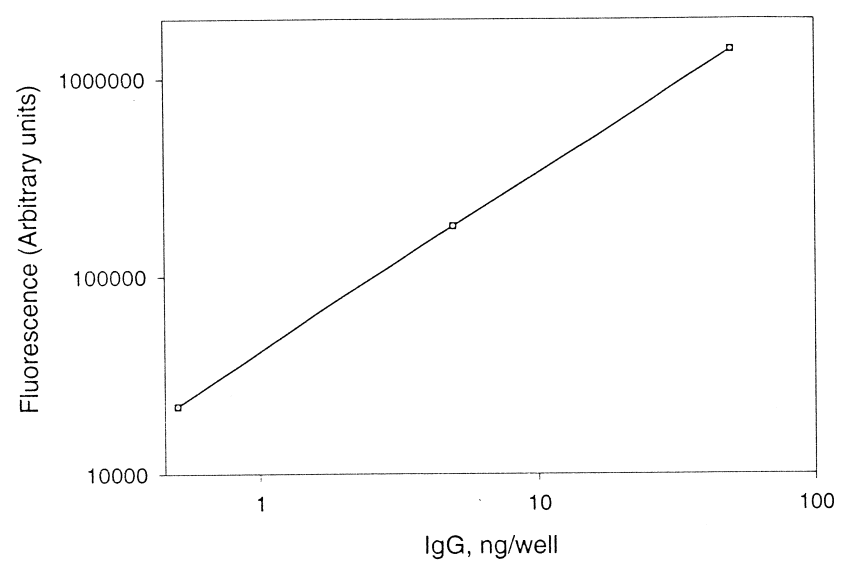

Figure 3 - Calibration curve for quantifying immobilized biotinylated mouse IgG. The background signal obtained with the zero calibrator (no IgG added) was $\sim 100$ arbitrary fluorescence units, subtracted from all the measurements.

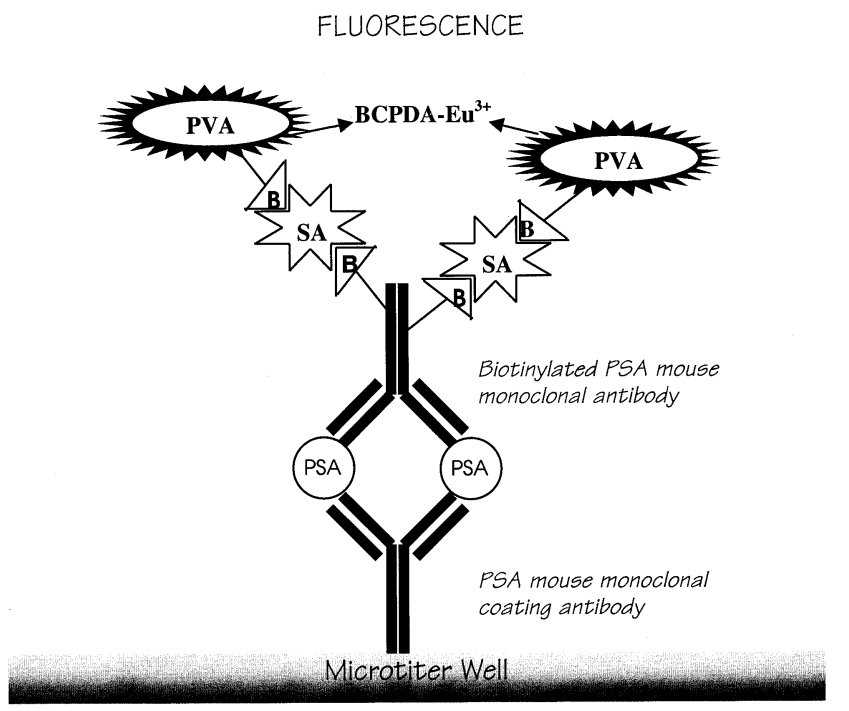

Figure 4 - Schematic representation of the $(\mathrm{SA})_{\mathrm{z}}-(\mathrm{b})_{\mathrm{x}}-$ PVA-(BCPDA) $)_{y}-\mathrm{Eu}^{3+}$ reagent and its utilization in the PSA immunoassay. $\mathrm{B}=$ biotin; $\mathrm{SA}=$ streptavidin; $\mathrm{PVA}=$ polyvinylamine. Each PVA molecule carries approximately 50-100 BCPDA-Eu ${ }^{3+}$ complexes.

5-10,000 ng/L (ten replicates per concentration) and was $27 \%$ at a PSA concentration of $1 \mathrm{ng} / \mathrm{L}$. When we compared this assay with assays using either enzymatically amplified time-resolved fluorometry $(8,11)$ or enzymatically triggered chemiluminescence with dioxetane substrates $(12,13)$ (same reagents and conditions), we obtained comparable results (similar signal to noise ratios and detection limits) but linearity was extended by one decade with the present method.

\section{Discussion}

The favorable properties of lanthanide chelates for time-resolved immunofluorometric assays have been previously reviewed $(2-5,14,15)$. Lan-

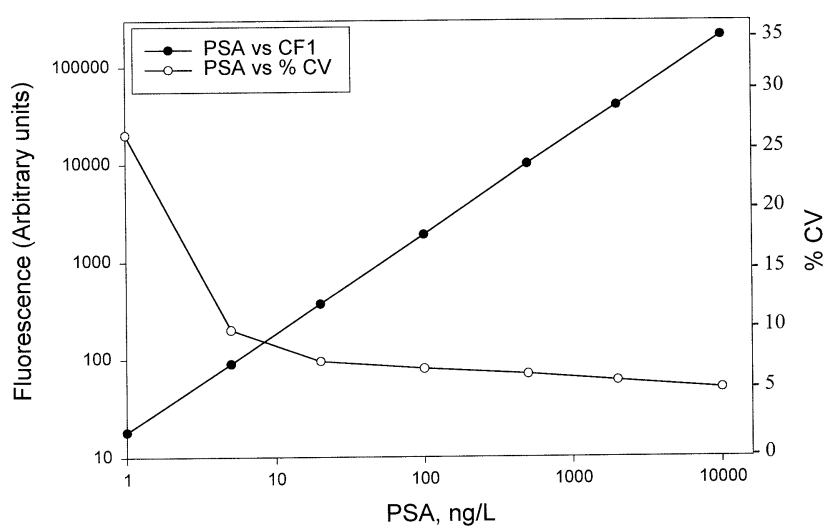

Figure 5 - Calibration curves for the PSA immunoassay using $(\mathrm{SA})_{\mathrm{z}}-(\mathrm{b})_{\mathrm{x}}-\mathrm{PVA}-(\mathrm{BCPDA})_{\mathrm{y}}-\mathrm{Eu}^{3+}$ conjugate as detection reagent. Plate was counted with a CyberFluor 615 Immunoanalyzer. Background was $\sim 80$ arbitrary units, subtracted from all measurements. Precision profile was based on ten replicates per concentration shown. The calibration curve was constructed using linear regression. 
thanide chelates can afford 100- to 1000 -fold better sensitivities in comparison to conventional fluorophors due to efficient background fluorescence rejection. Background rejection is achieved through the large Stokes shifts and the narrow emission bands of fluorescent lanthanide chelates as well as the application of the time-resolved fluorometric measurement (3).

We have previously shown that fluorescent lanthanide chelates are free of quenching when multiple labeling is used (10). We have taken advantage of this principle to devise macromolecular complexes multiply-labeled with lanthanide chelates for timeresolved immunofluorometric analysis. Although we were able to achieve picogram/milliliter sensitivities in model immunological assays, the major disadvantages with the previously described macromolecular complexes were their difficult preparation, their relative instability, and their very large molecular weight (bovine thyroglobulin was used as a carrier in these applications). The new complexes described here have the advantage of approximately threefold higher sensitivity, lower molecular weight (polyvinylamine has a molecular weight six times lower than that of bovine thyroglobulin while maintaining a higher density of potentially-labeled amino groups), less expensive, and ease of preparation by a simple reagent mixing step. The preformed complexes are stable over time and they are suitable for immunological assays using biotinylated antibodies as auxiliary reagents. For comparison of this reagent with the previously described reagent, please see Morton and Diamandis (10). We anticipate that the same complexes may have applications in nucleic acid hybridization assays but we did not test these assays.

We have previously described highly sensitive detection technologies based on enzymatically amplified time-resolved fluorescence using terbium chelates as labels (8). Furthermore, enzymaticallytriggered chemiluminescence is among the most sensitive detection techniques (16-18). When we compared our new reagents with these competing technologies in the same immunological assay for PSA, we found that all three methods afford similar detection limits. An advantage of the newly developed reagent is the ability to measure fluorescence directly from the solid phase. This may facilitate other applications including: (a) multi-analyte immunoassays based on spatially distributed immunodots (19); (b) microarray applications whereby the fluorescent spots can be scanned with laser excitation (20-22); (c) flow cytometry for detecting labeled cells; and (d) high throughput screening applications on high density microtiter plates or filters. Such applications are now under consideration.

Recently, other investigators succeeded in developing $\mathrm{Eu}^{3+}$ fluorescent chelators that are also suitable, like BCPDA, to solid-phase time-resolved immunofluorometry $(6,23-25)$. Others have devised successful homogenous time-resolved fluorometric immunoassays suitable for clinical applications (26, 27).

In conclusion, we describe here the development of a streptavidin-based detection reagent that can afford high sensitivity of detection and at the same time, is suitable for solid-phase fluorometric measurements. The reagent could find diverse applications in immunological, molecular, and cell-based assays and in high-throughput screening and microarrays.

\section{Acknowledgement}

We thank Anastasia Papanastasiou-Diamandi and Mohammad J. Khosravi for helpful discussions. This work was supported by grants to E.P. Diamandis from MDS Nordion, MDS Proteomics, the Medical Research Council of Canada University-Industry Program, and the Natural Sciences and Engineering Research Council of Canada University-Industry Program.

\section{References}

1. Hemmila I. Fluoroimmunoassays and immunofluorometric assays. Clin Chem 1985; 31: 359-70.

2. Diamandis EP, Christopoulos TK. Europium chelate labels in time-resolved fluorescence immunoassays and DNA hybridization assays. Anal Chem 1990; 62: 1149-57.

3. Diamandis EP. Immunoassays with time-resolved fluorescence spectroscopy: principles and applications. Clin Biochem 1988; 21: 139-50.

4. Diamandis EP. Time-resolved fluorometry in nucleic acid hybridization and Western blotting techniques. Electrophoresis 1993; 14: 866-75.

5. Hemmila I. Lanthanides as probes for time-resolved fluorometric immunoassays. Scand J Clin Lab Invest 1988; 48: 389-99.

6. Yuan J, Matsumoto K, Kimura H. A new tetradentate beta-diketonate-europium chelate that can be covalently bound to proteins for time-resolved fluoroimmunoassay. Anal Chem 1998; 70: 596-601.

7. Leif RC, Thomas RA, Yopp TA, et al. Development of instrumentation and fluorochromes for automated multiparameter analysis of cells. Clin Chem 1977; 23: 1492-8.

8. Christopoulos TK, Diamandis EP. Enzymatically amplified time-resolved fluorescence immunoassay with terbium chelates. Anal Chem 1992; 64: 342-6.

9. Evangelista RA, Pollak A, Allore B, Templeton EF, Morton RC, Diamandis EP. A new europium chelate for protein labeling and time-resolved fluorometric applications. Clin Biochem 1988; 21: 173-8.

10. Morton RC, Diamandis EP. Streptavidin-based macromolecular complex labelled with a europium chelator suitable for time-resolved fluorescence immunoassay applications. Anal Chem 1990; 62: 1841-5.

11. Diamandis EP, Yu H, Melegos DN. Ultrasensitive prostate-specific antigen assays and their clinical application. Clin Chem 1996; 42: 853-7.

12. Ferguson RA, Yu H, Kalyvas M, Zammit S, Diamandis EP. Ultrasensitive detection of prostate specific antigen by a time-resolved immunofluorometric assay and the Immulite ${ }^{\circledR}$ immunochemiluminescent third generation assay: potential applications in prostate and breast cancers. Clin Chem 1996; 42: 675-84. 
13. Melegos DN, Diamandis EP. Is prostate-specific antigen present in female serum? Clin Chem 1998; 44: 691-2.

14. Gudgin Dickson EF, Pollak A, Diamandis EP. Timeresolved detection of lanthanide luminescence for ultrasensitive bioanalytical assays. J Photochem Photobiol 1995; 27: 3-19.

15. Beltyukova SV, Egorova AV. Terbium chelates for fluorescence immunoassays. J Pharm Biomed Anal 1998; 18: 267-70.

16. Kricka LJ, Stanley PE. Bioluminescence and chemiluminescence literature. The 1996 literature: Part 1. $J$ Biolumin Chemilumin 1997; 12: 261-70.

17. Kricka LJ. Prospects for chemiluminescent and bioluminescent immunoassay and nucleic acid assays in food testing and the pharmaceutical industry. $J$ Biolumin Chemilumin 1998; 13: 189-93.

18. Kricka LJ. Nucleic acid detection technologies-labels, strategies, and formats. Clin Chem 1999; 45: 453-8.

19. Kakabakos SE, Christopoulos TK, Diamandis EP. Multianalyte immunoassay based on spatially distinct fluorescent areas quantified by laser-excited solid-phase time-resolved fluorometry. Clin Chem 1992; 38: 338-42.

20. Ekins RP. Ligand assays: from electrophoresis to miniaturized microarrays. Clin Chem 1998; 44: 201530 .
21. Ekins R, Chu FW. Microarrays: their origins and applications. Trends Biotechnol 1999; 17: 217-8.

22. Kricka LJ. Miniaturization of analytical systems. Clin Chem 1998; 44: 2008-14.

23. Yuan J, Wang G, Kimura H, Matsumoto K. Highly sensitive time-resolved fluoroimmunoassay of human immunoglobulin E by using a new europium fluorescent chelate as a label. Anal Biochem 1997; 254: 283-7.

24. Yuan J, Matsumoto K. Synthesis of a new tetradentate beta-diketonate-europium chelate and its application for time-resolved fluorimetry of albumin. J Pharm Biomed Anal 1997; 15: 1397-1403.

25. Kimura H, Yuan J, Wang G, Matsumoto K, Mukaida M. Highly sensitive quantitation of methamphetamine by time-resolved fluoroimmunoassay using a new europium chelate as a label. J Anal Toxicol 1999; 23: $11-6$.

26. Mathis G. Probing molecular interactions with homogeneous techniques based on rare earth cryptates and fluorescence energy transfer. Clin Chem 1995; 41: 1391-7.

27. Mathis G, Socquet F, Viguier M, Darbouret B. Homogeneous immunoassays using rare earth cryptates and time resolved fluorescence: principles and specific advantages for tumor markers. Anticancer Res 1997; 17: 3011-4. 